\title{
ARREGLO DE ANTENAS CON BAJO ACOPLAMIENTO MUTUO PARA SISTEMAS DE COMUNICACIONES INALÁMB RICAS MIMO-LTE
}

\section{ANTENNA ARRAY WITH LOW MUTUAL COUPLING FOR WIRELESS COMMUNICATION SYSTEMS MIMO-LTE}

\author{
PhD. Eduardo Rodríguez Araque*, PhD. Roberto G. Rojas-Teran** \\ * Universidad de Los Andes, Facultad de Ingeniería. \\ Carrera 1, No. \#18A-12, Bogotá, Cundinamarca, Tel.: (+571) 3394949, (+571) 3394999. \\ E-mail: e.rodriguez83@uniandes.edu.co \\ ** Ohio State University, ElectroScience Laboratory. \\ 205 Dreese Laboratories 2015 Neil Avenue, Columbus, Ohio 43210-1272, United State. \\ Tel.: (+1) 614 2926671, (+1) 6142927596. \\ E-mail: rojas-teran.1@osu.edu.
}

\begin{abstract}
Resumen: Este trabajo trata de un arreglo de antenas MIMO (multiple-input-multipleoutput) que opera en la banda de $2.6 \mathrm{GHz}$, una banda de Long Term Evolution (LTE), para sistemas de comunicaciones móviles inalámbricas $4 \mathrm{G}$. El arreglo de antenas consiste de 4 antenas compactas tipo patch sobre un substrato dieléctrico (PCB) de $125 \mathrm{~mm}$ x 62.5 $\mathrm{mm} \times 1.27 \mathrm{~mm}$. Modificaciones del plano de tierra (GND) junto con la ubicación sistemática y orientación de cada antena en la cara posterior al plano de tierra del PCB (Printed Circuit Board) juegan un rol muy importante en la reducción sustancial del acoplamiento mutuo, esto generalmente afecta el desempeño de los arreglos MIMO.
\end{abstract}

Palabras clave: Arreglo de antenas, modos característicos, correlación espacial, multipleinput-multiple-output (MIMO), acoplamiento mutuo.

\begin{abstract}
This work deals with a compact MIMO array that operates in the $2.6 \mathrm{GHz}$ for Long Term Evolution (LTE) band and wireless communication systems 4G. The array consists of four compact patch antennas on a dielectric substrate with total dimensions of $125 \mathrm{~mm}$ x $62.5 \mathrm{~mm} \times 1.27 \mathrm{~mm}$. Modifications on the ground plane along with systematic placement and orientation of each antenna on top of the substrate plays a key role in reducing the mutual coupling which normally degrades the MIMO array performance.
\end{abstract}

Keywords: Antenna array, characteristic modes, spatial correlation, MIMO, mutual coupling.

\section{INTRODUCCIÓN}

Los sistemas de arreglos MIMO son técnicas que nos permiten incrementar la tasa de transmisión de datos y la confiabilidad del enlace de comunicaciones cuando operan en ambientes ricos en scattering. Estas técnicas normalmente generan sub-canales paralelos poco correlacionados entre las antenas, lo que permite incrementar la capacidad del sistema medida en bit/s/Hz en varios órdenes de magnitud (Foschini y Gans, 1998).

Un factor importante que afecta fuertemente el performance de los sistemas MIMO es el acoplamiento mutuo entre las antenas del arreglo. Altos niveles de acoplamiento mutuo pueden causar incrementos en la correlación entre las señales recibidas de cada antena, y esto a su vez 
reduce el número de sub-canales paralelos independientes que el sistema MIMO puede llegar a generar (Ozdemir, Arvas, y Arslan, 2004). Por tanto, un alto rendimiento que puede ofrecer un sistema MIMO depende básicamente de: 1) las características multitrayecto (multipath) del canal de propagación, y 2) el acoplamiento mutuo entre las antenas del arreglo. Es poco probable que podamos modificar el canal de propagación; sin embargo, podemos realizar modificaciones sobre la estructura del arreglo y el GND para reducir el acoplamiento mutuo.

Existen técnicas para reducir el acoplamiento mutuo entre las antenas del arreglo; una forma es incrementar el espaciado entre las antenas. Esta técnica es factible cuando las antenas del arreglo son puestas en las estaciones base donde el espaciado puede ser incrementado sin limitaciones; sin embargo, si el arreglo de antenas es construido dentro de los pequeños teléfonos celulares, aumentar el espaciado se hace una tarea muy difícil de realizar y requiere de un trabajo complejo. Esto introduce nuevos retos a los diseñadores de antenas, conforme el espaciado entre las antenas del arreglo se convierte en una fuerte restricción. Diferentes diseños de antenas han sido propuestos para reducir el acoplamiento mutuo de los arreglos MIMO en pequeños teléfonos celulares. En (Karaboikis, Soras, y Makios, 2004), y (Mavridis, Sahalos, y Chryssomallis, 2006), algunas técnicas de inserción de slots en las antenas se desarrollan para lograr alto aislamiento (bajo acoplamiento mutuo). Estas técnicas pueden reducir la longitud de onda de la señal en la vecindad de las antenas y así, incrementar la separación eléctrica entre las antenas del arreglo (Karaboikis et al.,2004). Otros métodos usan slots T-shaped introducidos en el GND para reducir el acoplamiento mutuo como en Park, Choi, Park, Kim (2012). En (Li y Gong, 2008), slots T-shaped junto con inverted-L-shaped introducidos en el GND del PCB logran reducir fuertemente el acoplamiento mutuo.

Adicionalmente, técnicas de neutralización han sido utilizadas para incrementar el aislamiento puerto-a-puerto entre dos antenas través de una línea de transmisión conectada entre estas antenas. Estas técnicas son utilizadas con antenas PIFA (Chebini, Luxey, Diallo, Lethuc, y Staraj, 2009), y antenas monopolo impresas (See, Adb-Alhameed, Abidi, McEwan, y Excell, 2012). Existen esfuerzos utilizando estructuras EBG (electromagnetic band gap), las cuales suprimen las ondas de superficie (surface waves), y así se reduce el acoplamiento mutuo entre las antenas (Yang y Rahmat-Samii,
2003). La aplicación de estructuras tipo WAS (defected wall structures) es propuesta por Dadashzadeh, Dadgarpour, y Virdee (2011), junto con slits (slots) en el GND, separan eléctricamente dos antenas microstrip (patch) lo suficiente para proveer un aislamiento de $56 \mathrm{~dB}$. Todas estas técnicas, y otras como las planteadas por Mak, Rowell, y Murch (2008), y Yang, Liu, Zhou, y Cui (2012), han sido utilizadas para lograr las especificaciones de aislamiento que requieren los sistemas MIMO para obtener un alto rendimiento.

Aunque estas técnicas permiten un alto nivel de ais lamiento, y así obtener una muy baja correlación especial, es evidente que en muchas ocasiones debemos considerar las características e influencia del sustrato dieléctrico del PCB donde se construye el arreglo de antenas (chasis del teléfono celular), el cual llega a ser un factor crítico en el rendimiento de arreglos MIMO. Estas consideraciones no han sido rigurosamente analizadas en la literatura estudiada. Adicionalmente, la complejidad del diseño y fabricación requiere más atención y consideración.

Una técnica interesante y atractiva para entender el rol del chasis del teléfono sobre el rendimiento de MIMO es a través del análisis modal, denominado, análisis de Modos Característicos (Characteristic Modes, CM). Este análisis provee una visión más profunda de las características resonantes potenciales de un estructura mediante una rigurosa examinación de los modos naturales de la estructura bajo análisis, en nuestro caso el chas is del teléfono.

La teoría de CM fue introducida por Garbacz (1965), y más tarde refinada por Harrington y Mautz (1971). Básicamente, los CM's son modos de corriente reales que deben ser calculadas numéricamente, corrientes propias de la estructura con alguna forma arbitraria. Estos CM's forman un conjunto completo de Corrientes ortogonales sobre la estructura, así como un conjunto de patrones de radiación ortogonales. Esta propiedad nos permite usar la teoría de CM para diseñar un arreglo de antenas MIMO. Como resultado de este modelo de análisis, es posible seleccionar un conjunto de Corrientes características sobre el PCB para minimizar el acoplamiento mutuo entre las antenas del arreglo, y si es posible la reducción de la envolvente de la correlación.

El enfoque basado en CM considera que el chasis como estructura fundamental en el diseño, y por medio de la examinación del conjunto de las Corrientes características en el sustrato dieléctrico 
(PCB), permite al diseñador definir la posición de cada antena del arreglo MIMO.

Otra versión de esta teoría es denominada, red de modos característicos (Network Characteristic Modes, NCM), donde los modos son considerados como un conjunto de puertos sobre la estructura bajo estudio.

En este trabajo, proponemos un Nuevo diseño de arreglo MIMO para operar en la banda de $2.6 \mathrm{GHz}$ (4G-LTE). El arreglo de antenas consiste de 4 antenas patch compactas sobre un PCB. Modificaciones sobre el GND con slots junto con una sistemática ubicación y orientación de cada antena sobre la superficie superior del PCB juega un papel importante en el aumento considerable del aislamiento, y así la reducción de la correlación entre las antenas del arreglo.

Las modificaciones realizadas sobre el GND, la ubicación y orientación de las antenas sobre el arreglo fueron hechas basadas en la visión profunda que provee la teoría de modos característicos.

\section{DISEÑO DEL ARREGLO DE ANTENAS MIMO}

El objetivo en este trabajo fue diseñar un arreglo con un sustancial número de antenas dentro de un chasis compacto, y a su vez, tener un alto aislamiento entre las antenas. El diseño de la antena fue hecho tal que opera en $2.6 \mathrm{GHz}$, utilizando técnicas de miniaturización lo que permite diseñar antenas de tamaño pequeño usando algunas ideas de inserción de slots, como se puede observaren la Fig. 1.

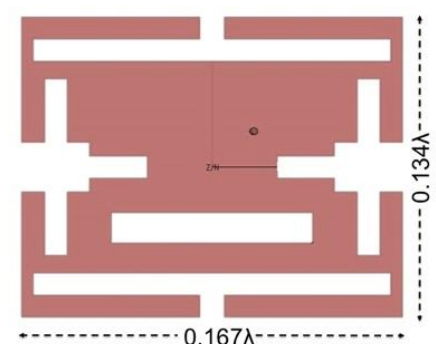

Fig. 1. Diseño de la antena tipo patch miniaturizada utilizando slots para operara 2.6 $\mathrm{GHz}$.

El siguiente paso es tomar cuatro antenas iguales a la de la Fig. 1, y ubicarlas sobre el sustrato (PCB), y obtener un muy bajo acoplamiento mutuo entre ellas. Para hacer esto, un procedimiento sistemático de modificación del GND y ubicación de las antenas es desarrollado. Cada paso de este procedimiento es explicado claramente en las siguientes secciones.

\subsection{Modificaciones del Plano de Tierra (GND)}

Iniciamos con una estructura base de referencia; donde las cuatro antenas tienen la misma orientación como se muestra en la Fig. 2.

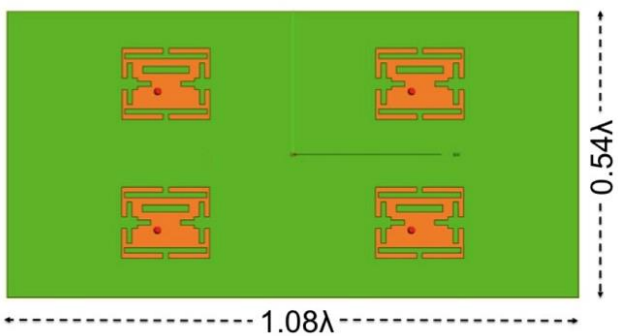

Fig. 2. La estructura base o de referencia donde se muestra las cuatro antenas operando a $2.6 \mathrm{GHz}$.

Aún, ninguna modificación sobre el GND del PCB se ha realizado. Para comenzar, modificamos la estructura con el fin de reducir el acoplamiento mutuo entre las antenas, un diseño de slots es introducido en el GND.

Estos slots ayudan a reducir el efecto producido por las ondas de superficie (surface waves) excitadas en el sustrato del PCB. Esta sistemática inserción de los slots sobre la estructura nos permiten seleccionar la estructura final del plano de tierra teniendo en cuenta la posición de cada slot así como el espaciado entre las antenas, con el fin de mantener el máximo espaciado posible entre ellas.

Al comparar la distribución de corriente total a 2.6 GHz de la estructura de referencia (Fig. 3a), y el GND con los slots (aun sin corrugar), se observa como la distribución de corriente decrece sustancialmente cuando los slots han sido introducidos. Esto significa que hay menos interacción entre las antenas y una reducción en el acoplamiento mutuo entre las antenas del arreglo es logrado.

Modificaciones adicionales son elaboradas mediante la inserción de pequeñas estructuras (pequeños slots rectangulares de $5 \mathrm{~mm}$ x $2.5 \mathrm{~mm}$ ) sobre los slots principales para lograr mayor reducción en el acoplamiento mutuo entre las antenas, como se muestra en la Fig. 3c. 

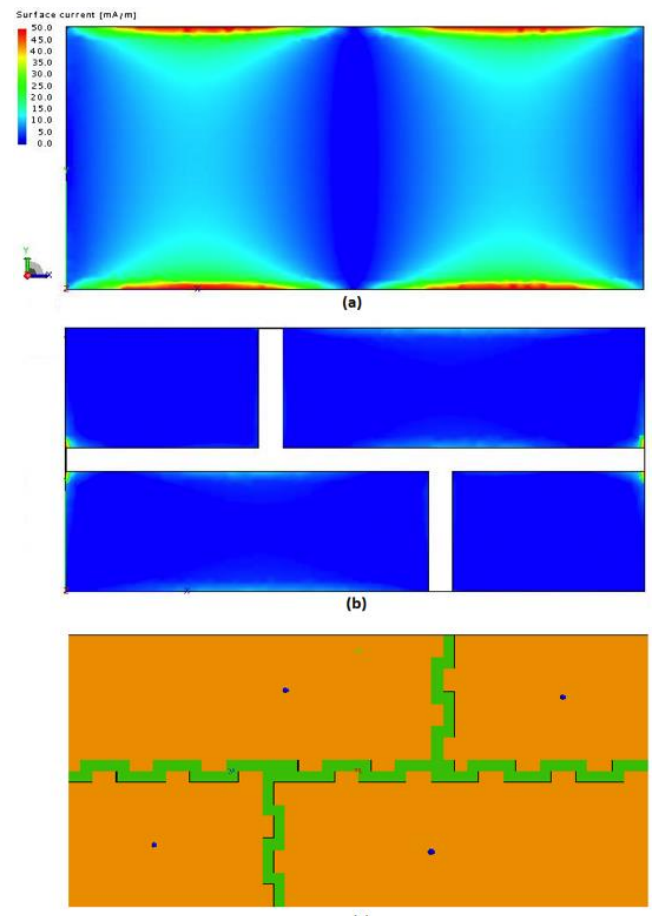

Fig. 3. Distribución de Corriente total en la frecuencia a $2.6 \mathrm{GHz}$ de (a) plano de tierra de referencia, (b) plano de tierra modificado con slots ( sin corrugar), $y(c)$ plano de tierra modificado con los slots rectangulares.

\subsection{Posición y orientación de las antenas}

Modificaciones adicionales son necesarias para obtener un mayor rendimiento, es decir más reducción en la correlación. La primera modificación se realiza sobre la posición de cada antena sobre el PCB. Inicialmente, cada antena se encuentra centrada en el correspondiente GND, luego modificaciones sobre la orientación de cada antena de realizado. Fue observado que una rotación específica de las antenas ayuda a mejorar la diversidad de polarización, así la correlación de las señales de cada antena del arreglo es reducida.

Una forma de obtener una orientación optima de cada antena en el arreglo es utilizando el análisis que ofrece CM. El espectro de eigenvalores NCM para las cuatro antenas del arreglo con los slots corrugados en el GND, y cada antena centrada en el correspondiente GND puede ser visto en la Fig. 4a. El espectro muestra el modo dominante de cada antena.

Los modos para las antenas \#1 y \#2 muestran una buena resonancia cercana a $2.6 \mathrm{GHz}$ (Fig. 4a), pero luego al realizar las modificaciones con la ayuda de CM muestra que todas las antenas están bien adaptadas a la frecuencia de $2.6 \mathrm{GHz}$, el resultado que genera las modificaciones sobre el comportamiento de los modos característicos y los parámetros $S_{11}$ (Pérdidas por Retorno) de cada antena puede ser visto en las Fig. 4b y Fig. 4d. Un análisis más detallado de $\mathrm{CM}$ fue desarrollado para obtener estos resultados.

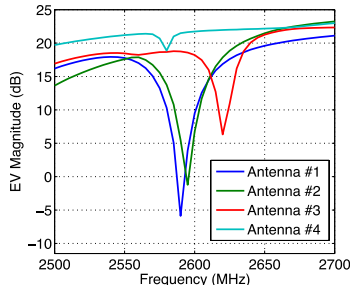

(a)

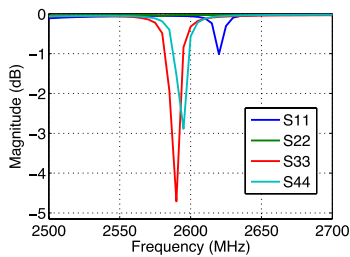

(c)

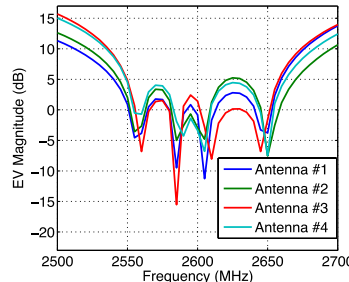

(b)

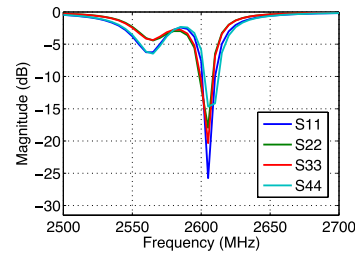

(d)
Fig. 4: Espectro de eigenvalores NCM del modo dominante de cada antena operando a $2.6 \mathrm{GHz}$. (a) Eigenvalores antes, $y(b)$ después de aplicar la rotación, (c) pérdidas por retorno $(R L)$ simuladas de cada antena antes, $y(d)$ después de la rotación (ver Fig. 5).

La Fig. 5 muestra la conformación final de cada antena sobre el PCB, y la geometría del GND.

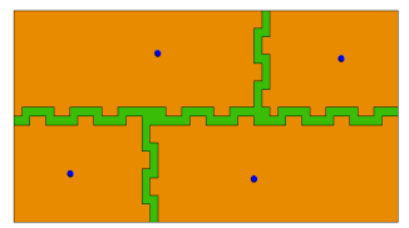

(a)

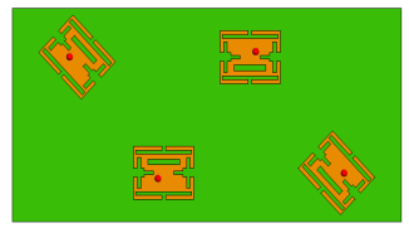

(b)

Fig. 5: Diseño final del arreglo MIMO.

(a) 4-antenna sobre el sustrato dieléctrico (PCB).

(b) GND modificado con slots corrugados (pequeños triángulos).

Cuatro antenas tipo patch miniaturizadas son impresas en la cara superior de un sustrato dieléctrico $\quad\left(\varepsilon_{r}=4.5\right.$ y $\left.\tan \delta=0.002\right) \quad$ con dimensiones de $125 \mathrm{~mm} \times 62.5 \mathrm{~mm} \times 1.27 \mathrm{~mm}$. 


\section{RESULTADOS Y DISCUSIÓN}

El rendimiento del arreglo de antenas MIMO es analizado a partir de resultados de mediciones y simulaciones de los parámetros S (scattering), acoplamiento mutuo o aislamiento, patrones de radiación, y correlación de envolvente.

\subsection{Parámetros S y Acoplamiento Mutuo}

La Fig. 6 muestra los resultados de simulaciones y mediciones de los parámetros $\mathrm{S}$ (respecto a una referencia de 50-ohm) para todas las antenas sobre el sustrato (PCB). Los parámetros $\mathrm{S}$ son medidos mientras las otras antenas son terminadas con cargas de 50-ohm. Los valores del parámetro de pérdidas por retorno $\left(S_{i i} i=j\right)$ muestran una muy concordancia o exactitud entre los simulados y los medidos. El acoplamiento mutuo medido $\left(S_{i j}, i \neq j\right)$ entre las antenas permanece por debajo de los -28 dB como se observa en la Fig. 6 b.

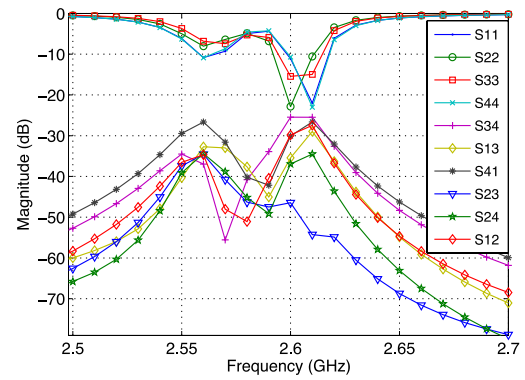

(a)

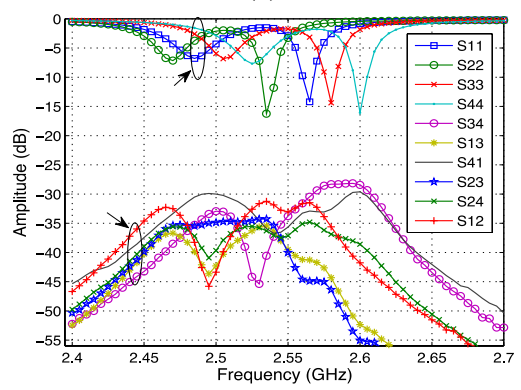

(b)

Fig. 6: Parámetros $S$ del arreglo propuesto. (a) Simulados. (b) Medidos

\subsection{Patrón de Radiación.}

Los patrones de radiación en 2D simulados y medidos a $2.6 \mathrm{GHz}$ de cada antena del arreglo son mostrados en la Fig. 7. Se puede observar que cada antena muestra un patrón de radiación diferente (diversidad del patrón); esto es una propiedad favorable que ayuda a reducir la correlación porque la distribución multi-trayecto del canal de
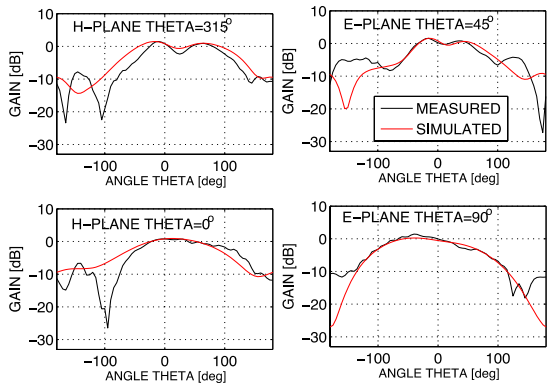

(a)
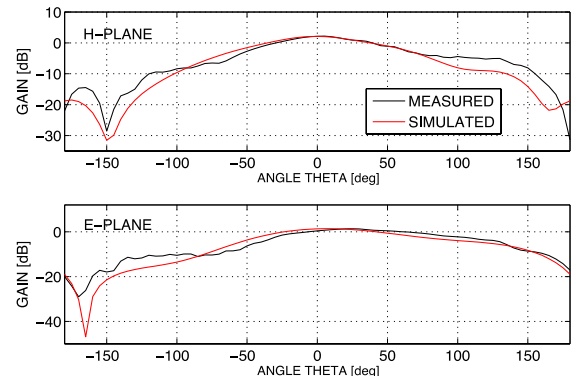

(b)
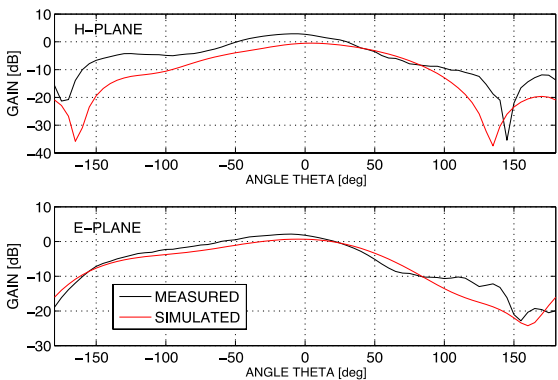

(c)
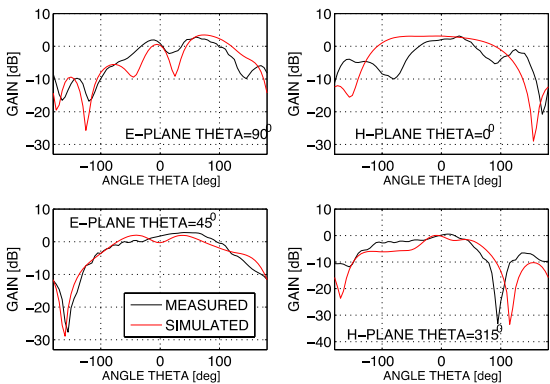

(d)

Fig. 7: Patrones de radiación en $2 D$ de cada antena medidos (línea negra), y simulados (línea roja), en la banda de 2.6 GHz. (a) Antena \#1. (b) Antena\#2. (c) Antena \#3. (d) Antena \#4. Se utilizó la cámara anecoica de ElectroScience Laboratory en Ohios State University, Columbus, Ohio, USA.

propagación recibida en cada antena llegará a ser diferente. Adicionalmente, los patrones de radiación medidos y simulados muestran una muy buena precisión. 
La máxima ganancia lograda es de $3.14 \mathrm{dBi}$. Las mediciones fueron desarrolladas excitando una antena mientras que las otras tres antenas fueron terminadas con cargas de 50-ohm.

\subsection{Evaluación de la Envolvente de Correlación}

Una métrica que provee importante información acerca del rendimiento de los arreglos de antenas para ser aplicados en sistemas MIMO es la correlación.

Esta métrica puede ser calculada a través de los parámetros $\mathrm{S}$ bajo la suposición que las señales que arriban a cada antena del arreglo son uniformemente distribuidas, es decir las direcciones de arribo de cada componente multipath tienen igual probabilidad de arribo (Blanch, Romeu y Corbella, 2003).

Los coeficientes de correlación para un arreglo de $\mathrm{N}$-antenas puede ser calculado como (Dama, Hussaini, Abd-Alhameed, Jones, McEwan, Sadeghpour, y Rodriguez, 2011). Se asume que las antenas del arreglo no tienen pérdidas.

$$
\rho_{i j, n}=\frac{\left|\sum_{n=1}^{N} S_{i, n}^{*} S_{n, j}\right|}{\prod_{k=(i, j)}\left[1-\sum_{n=1}^{N} S_{i, n}^{*} S_{n, k}\right]}
$$

Los resultados de los coeficientes de correlación para las cuatro antenas a través de los parámetros $S$ son mostrados en la Fig. 9.

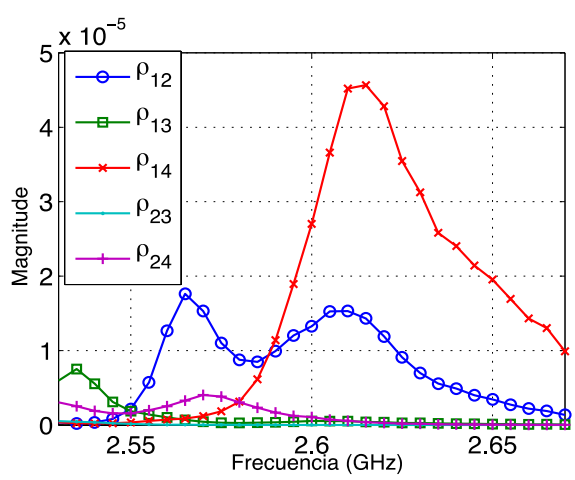

Fig. 8: Magnitud de los coeficientes de correlación calculados a través de los parámetros $S$.

\section{CONCLUS IONES}

El Arreglo de 4 antenas miniaturizadas propuesto es confiable y aplicable para operar en sistemas MIMO en la frecuencia de $2.6 \mathrm{GHz}$ ( banda LTE (AWS)). La modificación del GND con slots corrugados (pequeños triángulos metálicos) junto con una sistemática ubicación y orientación de las antenas (logando diversidad especial y de polarización) utilizando la visión más profunda que ofrece la teoría de Modos Característicos permitió que el arreglo de antenas MIMO construido tenga alto desempeño para ser utilizado en sistemas de comunicaciones inalámbricas. Adicionalmente, las características de radiación observadas en el arreglo propuesto tienen la habilidad de superar problemas causados por el atenuación multipath ya que los patrones generados proveen diversidad en su forma.

\section{REFERENCIAS}

Blanch, S., Romeu, J. \& Corbella, I. (2003). Exact representation of antenna system diversity performance from input parameter description. Electronics Letters, 39(9), pp. 705-707.

Chebihi, A., Luxey, C., Diallo, A., Le-Thuc, P. \& Staraj, R. (2009). A new method to increase the port-to-port isolation of a compact two-antenna umts system. Antennas and Propagation EuCAP2009, 3rd European Conference, pp. 1098-1101.

Dadashzadeh, G., Dadgarpour, A., \& Virdee, B. (2011). Mutual coupling suppression in closely spaced antennas. Microwaves and Antennas Propagation ofIET, 5(1), pp. 113-125.

Dama, Y. A., Hussaini, A., Abd-Alhameed, R. A., Jones, S. M., McEwan, \& Rodriguez, J. (2011). Envelope correlation formula for $(n, n)$ MIMO antenna array including power losses. Electronics, Circuits and Systems ICECS, 18th IEEE International Conference, pp. 5-12.

Garbacz, R. (1965). Modal expansions for resonance scattering phenomena. Proceedings of the IEEE, 53(8), pp. $324-357$.

Harrington, R., \& Mautz, J. (1971). Theory of characteristic modes for conducting bodies. Antennas and Propagation on IEEE Transactions, 19(5), pp. 622-628.

Karaboikis, M., Soras, C., Tsachtsiris, G., \& Makios, V. (2004). Compact dual-printed inverted-f antenna diversity systems for portable wireless devices. Antennas and Wireless Propagation Letters of IEEE, 3(1), pp. 9-14. 
Li, Z., Du, Z., \& Gong, K. (2008). A novel wideband printed diversity antenna for mobile phone application. Antennas and Propagation Society International Symposium of IEEE, pp. $1-4$.

Mak, A., Rowell, C., \& Murch, R. (2008). Isolation enhancement between two closely packed antennas. Antennas and Propagation of IEEE Transactions, 56(11), pp. 3411-3419.

Mavridis, G., Sahalos, J., \& Chryssomallis, M. (2006). Spatial diversity two-branch antenna for wireles devices. Electronics Letters, 42(5), pp. 266-268.

Ozdemir, M., Arvas, E., \& Arslan, H. (2004). Dynamics of spatial correlation and implications on mimo systems. Communications Magazine of IEEE, 42(6), pp. S14-S19.
Park, J., Choi, J., Park, J.Y., \& Kim, Y.-S. (2012) Study of a t-shaped slot with a capacitor for high isolation between mimo antennas. Antennas and Wireless Propagation Letters of IEEE, 11, pp. 1541-1544.

See, C. H., Abd-Alhameed, R., Abidin, Z., McEwan, N., \& Excell, P. (2012). Wideband printed mimo/diversity monopole antenna for wifi/wimax applications. Antennas and Propagation of IEEE Transactions, 60(4), pp. 2028-2035.

Yang, F., \& Rahmat-Samii, Y. (2003). Microstrip antennas integrated with electromagnetic bandgap (ebg) structures: a low mutual coupling design for array applications. Antennas and Propagation of IEEE Transactions, 51(10), pp. 2013-2024.

Yang, X. M., Liu, X. G., Zhou, X. Y. \& Cui, T.J. (2012). Reduction of mutual coupling between closely packed patch antennas using waveguided metamaterials. Antennas and Wireless Propagation Letters of IEEE, 11(2), pp. 389-391. 